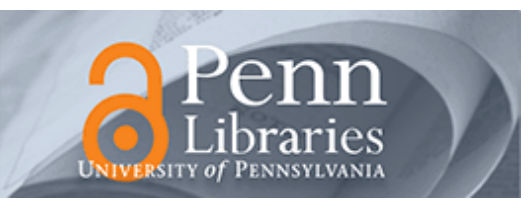

University of Pennsylvania ScholarlyCommons

October 2006

\title{
Simultaneous fluorometry and phosphorometry of Langendorff perfused rat heart: ex vivo animal studies
}

\author{
Mahsa Ranji \\ University of Pennsylvania \\ Dwight Jaggard \\ University of Pennsylvania, jaggard@seas.upenn.edu \\ Sofia Apreleva \\ University of Pennsylvania \\ Sergei Vinogradov \\ University of Pennsylvania \\ Britton Chance \\ University of Pennsylvania
}

Follow this and additional works at: https://repository.upenn.edu/ese_papers

\section{Recommended Citation}

Mahsa Ranji, Dwight Jaggard, Sofia Apreleva, Sergei Vinogradov, and Britton Chance, "Simultaneous fluorometry and phosphorometry of Langendorff perfused rat heart: ex vivo animal studies", . October 2006.

Copyright 2006 Optical Society of America, Inc. Postprint version. Published in Optics Letters, Volume 31, Issue 20, October 2006, pages 2995-2997.

This paper is posted at ScholarlyCommons. https://repository.upenn.edu/ese_papers/215

For more information, please contact repository@pobox.upenn.edu. 


\title{
Simultaneous fluorometry and phosphorometry of Langendorff perfused rat heart: ex vivo animal studies
}

\begin{abstract}
Fluorescence imaging of intrinsic fluorophores of tissue is a powerful method to assess metabolic changes at the cellular and intracellular levels. At the same time, exogenous phosphorescent probes can be used to accurately measure intravascular tissue oxygenation. Heart failure is the leading cause of death in America. A rat heart can potentially model the human heart to study failures or other abnormalities optically. We report simultaneous fluorescence and phosphorescence measurements performed on a rat heart. We have used two different optical systems to acquire fluorescence signals of flavoprotein and nicotinamide adenine dinucleotide-the two intrinsic fluorophores of mitochondria-and the phosphorescence signal of an intravascular oxygen probe to extract intracellular and intravascular metabolism loads, respectively.
\end{abstract}

\section{Keywords}

medical and biological imaging, medical optics instrumentation, spectroscopy, tissue diagnostics, fluorescence and luminescence

\section{Comments}

Copyright 2006 Optical Society of America, Inc. Postprint version. Published in Optics Letters, Volume 31, Issue 20, October 2006, pages 2995-2997. 
To be published in Optics Letters:

Title: $\quad$ Simultaneous fluorometry and phosphorometry of Langendorff perfused rat heart: ex vivo animal studies

Authors: Mahsa Ranji, dwight Jaggard, Sofia Apreleva, Sergei Vinogradov, and Britton Chance

Accepted: 25 July 2006

Posted: 3 August 2006

Doc. ID: 71096 


\title{
Simultaneous fluorometry and
}

\section{phosphorometry of Langendorff perfused rat}

\section{heart: ex vivo animal studies}

\author{
Mahsa Ranji, Dwight L. Jaggard \\ Complex Media Laboratory, Department of Electrical and \\ Systems Engineering, University of Pennsylvania \\ Philadelphia, Pennsylvania 19104 \\ Sofia V. Apreleva, Sergei A. Vinogradov, Britton \\ Chance \\ Department of Biochemistry and Biophysics, University of \\ Pennsylvania,
}

Philadelphia, Pennsylvania 19104 
Fluorescence imaging of intrinsic fluorophores of tissue is a powerful method to assess metabolic changes at the cellular and intracellular levels. At the same time, exogenous phosphorescent probes can be used to accurately measure intravascular tissue oxygenation. Heart failure is the leading cause of death in America. A rat heart can potentially model the human heart to study failures or other abnormalities optically. This paper reports simultaneous fluorescence and phosphorescence measurements performed on a rat heart. We have used two different optical systems to acquire fluorescence signals of Fp (Flavoprotein) and NADH (Nicotinamide Adenine Dinucleotide) - the two intrinsic fluorophores of mitochondria - and the phosphorescence signal of an intravascular oxygen probe to extract intracellular and intravascular metabolism loads respectively.

(c) 2006 Optical Society of America

OCIS codes: $300.6280,170.6510,170.3880,170.3890$

Optical imaging of tissue is a noninvasive and well-studied technique that has been widely used in biomedical applications. Optical methods can be employed as a diagnostic or therapeutic tool for ex vivo small animal studies and possibly for human disease diagnosis and therapy. Heart failure is one of the leading high-risk diseases that can be optically investigated. In this regard, the correlation of intracellular and vascular oxygen concentration in a rat heart could be an important factor in vascular disease diagnosis and progression. In 
addition, it would be helpful to observe the oxygenation signals of the metabolic states of tissue in open-heart surgeries. The Langendorff perfusion method has been used by many research groups for myocardial ischemic border zones, ${ }^{1}$ regional ischemia in hypertrophia, ${ }^{2}$ and heart muscle oxygenation ${ }^{3}$ studies. In previous studies the NADH images of the ischemic myocardium have clearly shown the LAD (left anterior descending artery) infarct borders ${ }^{3,4}$ and have been verified by microchemistry. ${ }^{5}$ Here we add a flavoprotein signal to obtain a ratio metric redox. In this study, cardiac mitochondrial intrinsic fluorescence signals, Fp and NADH, and phosphorescence of an exogenous oxygen probe (Oxyphor G3) ${ }^{6}$ are investigated. While fluorometery has been used in this study to collect signals related to the tissue oxygenation state, a phosphorescent probe, dissolved in blood plasma, served as an indicator of oxygen levels in the microvasculature.

Our fluorometer is a mobile optical-electrical apparatus that collects fluorescence signals through a $3 \mathrm{~mm}$ tip light guide. The light source is a brightline mercury arc lamp whose light is filtered at 4 different wavelengths by passing through an air turbine rotating filter wheel at a speed of $60 \mathrm{~Hz}$. Consequently, up to four signals can be multiplexed to a photodetector in order to make four-wavelength channel optical measurements of tissue metabolism. ${ }^{7}$ The light intensity that is incident on tissue at the fiber tip is $\sim 3 \mu w$. This instrument has the advantages of high speed time-sharing, mobility, simplicity, and high-resolution. Figure 1 shows the schematic of the fluorometer in acquiring spectroscopic heart data. For cardiac fluorometery experiments, two channels are used for FP and NADH excitation: $420 \mathrm{~nm}$ and $365 \mathrm{~nm}$, respectively (interference filters: 440DF20, 365HT25). The emitted fluorescent 
signals at $520 \mathrm{~nm}$ and $440 \mathrm{~nm}$ for FP and NADH, respectively, are detected through a PMT (photomultiplier tube) after passing through bandpass filters (interference filters: 525DF50, 455DF70). The signal is then digitized and displayed. Hence, the NADH and FP signals are monitored online using surface fluorescence in an isolated working rat heart under different heart rates.

Measuring intravascular oxygenation by phosphorescence quenching is a well known technique, widely used to study biological systems. ${ }^{8}$ Oxygen quenches the luminescence of exogenous phosphorescent probes dissolved in the blood. The concentration of oxygen is inversely proportional to phosphorescence lifetime (T) in accordance with the Stern-Volmer relationship

$$
T / T_{0}=1+K_{q} * T_{0} *\left[O_{2}\right]
$$

where $K_{q}$ and $T_{0}$ are probe related constants. In this study we used a new phosphorescent probe, Oxyphor G3 - Pd-tetrabenzoporphyrin-dendrimer, peripherally modified with oligoethyleneglycole residues. Oxyphor G3 is confined to the microcirculation and does not diffuse out into the interstitial space. The excitation wavelength for Oxyphor G3 is $635 \mathrm{~nm}$ and its phosphorescence maximum is at $810 \mathrm{~nm} . K_{q}$ of $170 \mathrm{~mm} \mathrm{Hg}^{-1} \mathrm{~s}^{-1}$ and $T_{0}=270 \mathrm{msec}$ make Oxyphor G3 well suited for oxygen measurements throughout the range of physiological oxygen concentrations. Due to the position of the excitation band far in the red region (635 nm), a relatively deep tissue volume can be sampled using surface excitation of Oxyphor G3. Consequently, in our experiments phosphorescent signals from Oxyphor G3 were so strong 
that the optical fiber (see below) could be positioned at a distance from the heart, and no contact was required. Phosphorescence measurements were performed using a digital phosphorometer. ${ }^{9}$ In brief, the excitation source is a $3 \mathrm{~W} \operatorname{LED}\left(\lambda_{\max }=635 \mathrm{~nm}\right)$ and the detector is an APD (3 $\mu$ sec rise time). The excitation and the emission are conducted to and from the instrument by a $3 \mathrm{~mm}$ bifurcated light guide. The emission is filtered through a long-pass (695 nm cut-off) filter and focused on the APD by a spherical lens. The sample is excited by a pulse of light (time-domain operation), and the phosphorescence decay is digitized and analyzed to give the phosphorescence lifetime and oxygen concentration.

Vascular oxygen concentration measured by phosphorometry is closely related to the cellular oxygenation state inferred from fluorometry. Figure 2 shows the two probes for fluorescence and phosphorescence simultaneous measurements. To minimize environmental light, the room was dark and a black cloth surrounded the system after the probes were positioned. Oxyphor G3 was added to the buffer which was continuously passing through the heart.

Although fluorescence spectroscopy of myocardium tissue is well-studied ${ }^{10,1,11}$ and $\mathrm{O}_{2}$ probe monitoring is a known technique, simultaneous measurements of fluorescence and phosphorescence have only been correlated to a limited extent. This experiment serves as a control to correlate intracellular $\mathrm{O}_{2}$ concentration, which is deducted from mitochondrial redox states, and vascular $\mathrm{O}_{2}$ concentration, which results from $\mathrm{O}_{2}$ probe monitoring. This can be useful in studies of defective oxygen delivery, apoptosis, open chest surgery tissue oxygenation monitoring, cancerous tissue studies, and oxygen endoscopy. In this regard, the correlation of intracellular and vascular oxygen concentration in a rat heart could be an 
important study in vascular disease diagnosis and progression.

The apparatus for crystalloid perfusion is Langendorff System from ADInstruments using Krebs Buffer (KHB) as perfusate. The gas used for crystalloid experiment is $95 \%$ oxygen, $5 \%$ carbon dioxide. Sprague-Dawley rats of approximately 300 grams are anesthetized with a mixture of ketamine and acepromazine $(1.0 \mathrm{mg} / \mathrm{kg}$ and $0.1 \mathrm{mg} / \mathrm{kg}$, respectively). After anesthesia, the hearts are rapidly excised and placed in ice-cold perfusate. Once perfused with a buffered, oxygenated solution the heart will beat spontaneously, but during this experiment it is paced using an external stimulator with a stimulus that exceeds the natural cardiac pacemaker rate for different metabolic activities. Figure 3 displays the fluorescence and the phosphorescence signals of myocardium, which are measured simultaneously in different heart situations. As can be seen, the protocol starts at a heart rate of $200 \mathrm{bpm}$ with oxygen bubbling in the buffer and continues with a systematic hypoxia for less than five minutes by stopping the oxygen flow. Hence, the fluorescent signal of NADH increases with the stop of oxygen flow due to the reduction of this fluorophore that is fluorescent in reduced form. On the other hand, FP decreases at this interval because it also gets reduced while it is more fluorescent in oxidized form. After this, the heart rate is increased by a stimulator to 300bpm and $400 \mathrm{bpm}$, and the same procedure of first section is executed. In the exact range of these time events, which are emphasized with two vertical dashed lines, the phosphorescence signal shows behavior that is consistent with the experimental protocol of flow and stop-flow of $\mathrm{O}_{2}$. This suggests that intracellular and vascular tissue oxygen levels in each time slot are highly correlated, though the trend of each fluorophore is quite different. The trend of the NADH 
graph has a negative slope while for FP it has a positive slope which reflects a highly oxidized myocardium due to global ischemia and probably initiation of apoptosis. On the other hand there is a distinguishable difference in the exponential rate of NADH increase versus the abrupt decrease of phosphorescence. This can suggest different sensitivities of these methods to oxygen sensing.

In conclusion, any abnormality in oxygen delivery, cardiac respiratory chain, and hypertrophied hearts could suggest a decorrelation of fluorescence-phosphorescence traces . Therefore, the $2 \mathrm{D}$ fluorescence or phosphorescence mapping provided by previous researchers ${ }^{2,4,3}$ and ourselves validates the hypothesis that direct fluorometry of mitochondrial signals and phosphorometry of vascular oxygen concentration are superior methods. These methods might be not only the indicator of hypoxia but also an indicator of irreversible damage to the myocardium in terms of activating apoptosis which causes a high redox ratio. ${ }^{12}$ Finally, these noninvasive optical techniques can be advantageous in assessing tissue oxygenation on site in open-heart surgery.

The author would like to thank A. Mu and Dr. K. Margulies for the rat heart facilities, and C. Alter for experimental set up help. This work was supported in part by grant NIH CA096016. Mahsa Ranji's email is: mahsa@seas.upenn.edu.

\section{References}

1. A.H. Harken, C.H. Barlow, W.R. Harden, B.Chance, American J. of Cardiology 42, (1978) 
2. J.F. Ashruf, C. Ince, and H.A. Bruining, Am J Physiol Heart Circ Physiol 277, (1999).

3. C.H. Barlow, E. Bailey, K.A. Kelly, JJ. Kelly, Plentum Press, 1998.

4. O.G. Bjornsson, K. Kobayashi, and J.R. Williamson, Am. Physiol Soc, (1987).

5. B. Chance, B. Schoener, K. Krejci, H. Russmann, W. Wesemann, H. Schnitger, Th. and Bucher, Biochem. Zeit, 341(1965).

6. I. B. Rietveld, E. Kim and S. A. Vinogradov, Tetrahedron, 59, (2003).

7. B. Chance, D. Mayer, N. Graham, V. and Legallais, Rev. Sci. Instru., 41, (1970).

8. D.F. Wilson, S.A. Vinogradov, Handbook of Biomedical Fluorescence, Marcel Dekker, 2003.

9. S. A. Vinogradov, M. A. Fernandez-Seara, B. W. Dugan and D. F. Wilson, Rev. Sci. Instrum., $\mathbf{7 2},(2001)$.

10. F.W. Heineman and R.S. Balaban, Annu. Rev. Physiol. 42, (1990).

11. F.W. Heineman, R.S. Balaban, Am. J. Physiol. 264, (1993)

12. M. Ranji, D.L. Jaggard, B. Chance, Proc. of SPIE 6087, (2006). 
Figure captions:

1) Two-wavelength time-shared fluorometer in rat heart fluorescence measurements and digital time-domain phosphorometer to monitor intravascular pO2

2) Simultaneous fluorescence-phosphorescence measurements on a rat heart (upper center)

3) NADH, FP Fluorescence and $\mathrm{O}_{2}$ probe phosphorescence time events 


\section{List of Figures}

Fig. 1 Two-wavelength time-shared fluorometer in rat heart fluorescence measurements and digital time-domain phosphorometer to monitor intravascular pO2

Fig. 2 Simultaneous fluorescence-phosphorescence measurements on a rat heart (upper center)

Fig. 3 NADH, FP Fluorescence and $O_{2}$ probe phosphorescence time events 

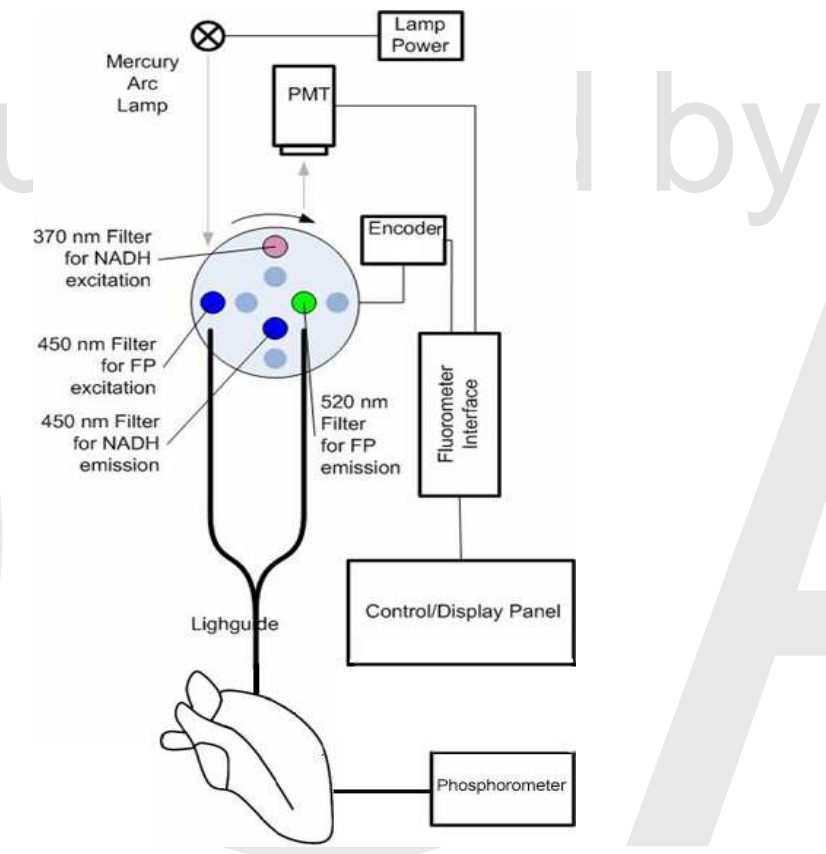

Fig. 1. Two-wavelength time-shared fluorometer in rat heart fluorescence measurements and digital time-domain phosphorometer to monitor intravascular pO2 

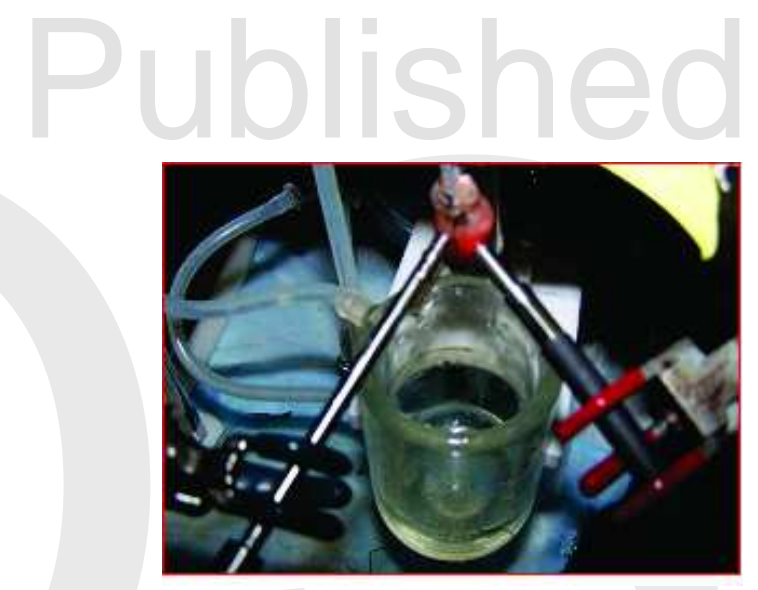

Fig. 2. Simultaneous fluorescence-phosphorescence measurements on a rat heart (upper center) 


\section{Time events}

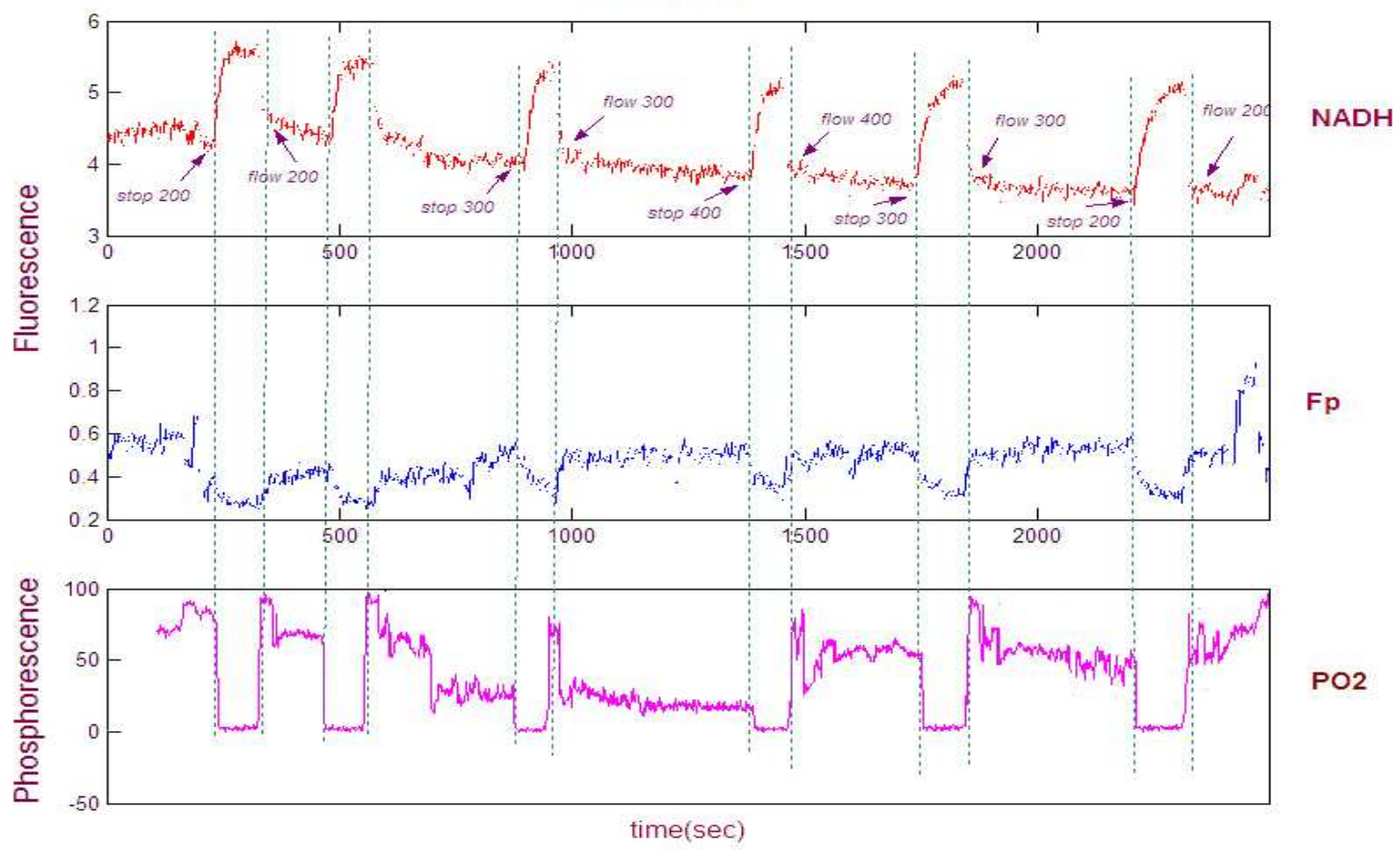

Fig. 3. NADH, FP Fluorescence and $\mathrm{O}_{2}$ probe phosphorescence time events 